\title{
A population-based study of breast implant illness
}

\author{
David A. Magno-Padron ${ }^{1}$, Jessica Luo ${ }^{1}$, Terry C. Jessop ${ }^{2}$, Jared W. Garlick ${ }^{1}$, Joanna S. Manum ${ }^{1}$, \\ Gentry C. Carter ${ }^{3}$, Jayant P. Agarwal ${ }^{1}$, Alvin C. Kwok $^{1}$ \\ ${ }^{1}$ Division of Plastic Surgery, Department of Surgery, University of Utah School of Medicine, Salt Lake City, UT; ${ }^{2}$ University of Utah School of \\ Medicine, Salt Lake City, UT; ${ }^{3}$ Department of Population Health Sciences, University of Utah School of Medicine, Salt Lake City, UT, USA
}

Background Despite evidence supporting the safety of breast implants, some women associate their implants with adverse health effects and have called this syndrome "breast implant illness." We sought to characterize breast implant illness symptoms and to report how implant removal affects their symptoms.

Methods An anonymous 20 question survey was administered to the Facebook group: "UTAH Breast Implant IIIness" to characterize the symptoms these women attributed to their breast implants. Several questions allowed us to evaluate how implant removal affected women's symptoms.

Results Of the 182 respondents, $97 \%$ report that implants negatively affect their health and 95\% identify these symptoms with breast implant illness. Ninety-six percent of respondents had implants placed for cosmetic reasons and $51 \%$ had silicone implants. The most common symptoms associated with breast implant illness are brain fog (95\%), fatigue (92\%), joint pain $(80 \%)$, and hair loss $(74 \%)$. Sixty percent of respondents learned about breast implant illness from family/friends and/or social media platforms (56\%), 40\% of respondents had their implants removed, and 97\% report relief of their symptoms post-removal (23\% complete, $74 \%$ partial). Following explantation, there was a significant improvement in all but one reported symptom. An association was found between the number of symptoms reported prior to explantation and the number of symptoms resolving following explantation.

Conclusions Breast implant illness is a syndrome characterized by fatigue, decreased focus, hair loss, and joint pain after the placement of breast implants. Nearly all patients report improvement of symptoms after implant removal. Significant efforts should be made to better understand breast implant illness and its etiology.

Keywords Breast prosthesis implantation / Cosmetic surgery / Surgery, plastic / Adverse effects
Correspondence: Alvin C. Kwok Division of Plastic Surgery, Department of Surgery, University of Utah School of Medicine, 30 North 1900 East, 3B400, Salt Lake City, UT 84132, USA

Tel: +1-801-581-7719

Fax: +1-801-581-5794

E-mail: Alvin.kwok@hsc.utah.edu
This article was presented at Mountain West Society of Plastic Surgeons on February 27, 2020, in Snowmass Village, CO, USA.

We wish to thank our patient representatives, Karen Hardy and Kara Fowers, who served as liaisons between the authors and the "UTAH Breast Implant IIIness" Facebook group, while also assisting with survey development and the distribution of the survey to the group.

Received: October 29, 2020 - Revised: April 16, 2021 • Accepted: May 25, 2021

pISSN: 2234-6163 • elSSN: 2234-6171 • https://doi.org/10.5999/aps.2020.02117• Arch Plast Surg 2021;48:353-360

\section{INTRODUCTION}

Breast implant illness (BII) is a term recently popularized by women advocacy groups and refers to the compilation of symptoms considered to be imparted by breast implants. The symp- toms attributed to BII are extensive and involve nearly every system in the body $[1,2]$. With over 300,000 breast augmentations and over 80,000 prosthetic-based breast reconstructions performed in 2018, the attention BII has received is not unfounded [3]. 
The debate regarding the possibility of breast implants causing systemic symptoms has been present since the 1960s, a theory which stemmed from an author's concept of human adjuvant disease; it refers to human exposure to a foreign object that causes autoimmune or rheumatic diseases $[4,5]$. The theory was gradually introduced to the public during the 1980s, later gaining the attention of the Food and Drug Administration (FDA), which in part led to the removal of silicone implants from the market in 1992 [6,7].

While many publications attempted to establish a link between silicone implants and autoimmune, connective tissue, and rheumatic diseases, most were unable to establish a definitive association [8-10]. Silicone implants returned to the market in 2006 with the stipulation that implant manufacturers must collect long-term outcomes data to evaluate their safety [11].

Coroneos et al. [12] published their analysis of the data gathered from the post-approval studies in 2018, concluding that silicone implants were associated with increased rates of specific connective tissue disease, rheumatic diseases, and other deleterious outcomes. Although others criticized their conclusions, they garnered considerable media attention and reignited the concerns around silicone implants [13-15].

A growing number of patients report that they suffer from BII. Many use social media as an outlet to share their symptoms, experiences, and search for a cure. Unfortunately, there is a limited understanding of what defines BII. This manuscript aims to characterize BII, and present patient-reported outcomes prior to and following explantation.

\section{METHODS}

We administered an anonymous online survey to a private Facebook group called "UTAH Breast Implant Illness" [16]. This private group consists of 1,281 members with daily participation. When coupled with Utah's substantial per capita rate of breast implant placement, we considered this group to be an ideal population to analyze. The survey consisted of 20 questions that sought to characterize the types of implants placed, adverse health-related symptoms attributed to breast implants, autoimmune conditions, and outcomes following explantation (see survey, Supplemental Material 1). To maintain privacy and Health Insurance Portability and Accountability Act (HIPAA) compliance, the reporting of age was omitted from our survey. The survey and study design were reviewed by our hospital's review board and was given exempt status.

We computed unweighted proportions of implant types, textures, and the plane of placement (subglandular vs. submuscular) among those who indicated they had BII symptoms and those who pursued explantation. A modified Delphi panel consisting of a board-certified plastic surgeon, a plastic surgery resident, a medical student, a physician's assistant, and two patient advocates was used to identify potential symptoms of BII, ultimately selecting 32 different symptoms to be included in the survey. Questions were presented such that women were allowed to select the symptoms that they attribute to their implants, and the temporal relationship these symptoms had with implantation and explantation. Respondents were able to select any number of these symptoms, thus allowing the authors to calculate the prevalence of each symptom in the overall cohort.

In those who pursued explantation, we calculated means and standard deviations for the number of symptoms each respondent reported before and after explantation. McNemar exact test for marginal homogeneity was employed to elucidate whether explantation had a significant effect on symptom improvement for each of the 32 symptoms.

In those who reported symptom improvement following explantation, the relationship between the number of symptoms reported prior to explantation and the number of symptoms that improved following explantation was analyzed. Univariate linear regression was applied both to those women who reported complete and partial resolution of symptoms. This allowed us to elucidate whether the number of symptoms reported prior to explantation could predict the number of symptoms expected to improve following explantation.

Respondents were allowed to report histories of autoimmune disease, what diagnosis they carry, and the temporal relationship their diagnosis had with implant placement. Diagnoses were self-reported and in free-text format. Confirmation of their diagnosis was not obtained.

\section{RESULTS}

The survey was distributed to the "UTAH Breast Implant Illness" Facebook group. There were 182 total respondents out of 1,281 group members (response rate, $14.2 \%$ ), the majority of whom had implants placed for cosmetic reasons (96\%).

Ninety-seven percent of women described an implant-related problem that began greater than 2 years following implantation. The most commonly reported implant-related problem was BII $(95 \%, \mathrm{n}=168)$, followed by capsular contracture and change in implant position (each $n=53$ ). Most women who reported BII learned of it from friends or family (60\%), social media (56\%), or the internet (50\%), while only three reported learning of it from a plastic surgeon. In those reporting BII, the most common type, texture, and position of the implant were silicone (51\%), smooth (76\%), and sub-pectoral positioning (93\%), re- 
spectively. Twelve respondents each were unaware of their implant type and texture, while four women were unaware of their plane of placement (Table 1).

Seventy women (42\%) who reported BII pursued explantation, only two of whom underwent implantation for reconstructive reasons. Ninety-seven percent of this cohort reported improvement of symptoms following explantation. Twenty-three percent $(n=16)$ of women experienced complete resolution of symptoms after implant removal and reported a mean average of 20.5 symptoms prior to explantation. Seventy-four percent $(n=52)$ of women reported only a partial resolution of symptoms following explantation. Of these respondents, they reported a mean average of 18 symptoms before implant removal while also reporting a mean average of 10.5 symptoms following explantation. Only two respondents had no improvement in their symptoms following explantation (Table 2).

\section{Autoimmune disease}

A history of autoimmune diagnoses was reported by $50 \%$ of our overall cohort. The majority (92\%) reported that their diagnosis occurred after implantation, with $65 \%$ reporting disease onset greater than 2 years after implantation.

\section{Symptoms}

To better characterize BII, respondents were asked to report all symptoms they personally attribute to BII. Thirty-two different symptoms were reported by 168 women with BII; those with

Table 1. Implant characteristics

\begin{tabular}{lccc}
\hline Characteristics & Overall $(\mathrm{n}=182)$ & BII $(\mathrm{n}=168)$ & Explant $(\mathrm{n}=70)$ \\
\hline Implant type & & & \\
$\quad$ Saline & $75(41)$ & $71(42)$ & $27(39)$ \\
Silicone & $90(49)$ & $85(51)$ & $41(59)$ \\
Unknown & $17(9)$ & $12(7)$ & $2(3)$ \\
Implant texture & & & \\
$\quad$ Textured & $29(16)$ & $29(17)$ & $15(21)$ \\
Smooth & $136(75)$ & $127(76)$ & $53(76)$ \\
Unknown & $17(9)$ & $12(7)$ & $2(3)$ \\
Implant position & & & \\
Over muscle & $7(4)$ & $7(4)$ & $4(6)$ \\
Under muscle & $169(93)$ & $157(93)$ & $64(91)$ \\
Unknown & $6(3)$ & $4(2)$ & $2(3)$ \\
\hline
\end{tabular}

Values are presented as number (\%).

Bll, breast implant illness. the highest prevalence are as follows: brain fog (decreased ability to concentrate) $(95 \%, \mathrm{n}=159)$, fatigue $(92 \%, \mathrm{n}=155)$, joint pain $(80 \%, n=135)$, and hair loss $(74 \%, n=124)$. Other than early menopause $(16 \%, n=29)$, all other symptoms had a prevalence of $20 \%$ or greater (Table 3 ).

We investigated those symptoms that were present before and after explantation in those who pursued the removal of their breast implants. Preoperatively, both fatigue and brain fog again

Table 3. Prevalence of symptoms before and after explantation

\begin{tabular}{|c|c|c|c|c|}
\hline \multirow[b]{2}{*}{ Symptoms } & \multirow{2}{*}{$\begin{array}{l}\text { Overall } \\
\text { Bll } \\
\text { cohort }\end{array}$} & \multicolumn{3}{|c|}{ Explantation cohort } \\
\hline & & $\begin{array}{l}\text { Explanters } \\
\text { with } \\
\text { symptoms }\end{array}$ & $\begin{array}{c}\text { Explanters } \\
\text { with symptom } \\
\text { improvement }\end{array}$ & P-value \\
\hline Fatigue & $155(92)$ & $66(94)$ & $50(76)$ & $<0.001$ \\
\hline Headache & $103(61)$ & $44(63)$ & $35(80)$ & $<0.001$ \\
\hline Anxiety & $117(70)$ & $51(73)$ & $39(76)$ & $<0.001$ \\
\hline Depression & $107(64)$ & $48(69)$ & $36(75)$ & $<0.001$ \\
\hline Vertigo & $78(46)$ & $34(49)$ & $28(82)$ & $<0.001$ \\
\hline Brain fog & $159(95)$ & $66(94)$ & $48(73)$ & $<0.001$ \\
\hline Poor sleep & $114(68)$ & $46(66)$ & $37(80)$ & $<0.001$ \\
\hline Changes in vision & $115(68)$ & $51(73)$ & $28(55)$ & $<0.001$ \\
\hline Changes in hearing & $69(41)$ & $29(41)$ & $14(48)$ & $<0.001$ \\
\hline Joint pain & $135(80)$ & $58(83)$ & $45(78)$ & $<0.001$ \\
\hline Muscle pain & $111(66)$ & $47(67)$ & $31(66)$ & $<0.001$ \\
\hline Numbness & $112(67)$ & $51(73)$ & $38(75)$ & $<0.001$ \\
\hline Cold/tingling limbs & $75(45)$ & $37(53)$ & $24(65)$ & $<0.001$ \\
\hline Breast discomfort & $113(67)$ & $45(64)$ & 41 (91) & $<0.001$ \\
\hline Rashes & $74(44)$ & $37(53)$ & $26(70)$ & $<0.001$ \\
\hline Hair loss & $124(74)$ & $56(80)$ & $33(59)$ & $<0.001$ \\
\hline Dry skin & $114(68)$ & $52(74)$ & $33(63)$ & $<0.001$ \\
\hline Dry eyes & $95(57)$ & $41(59)$ & $24(59)$ & $<0.001$ \\
\hline Easing bruising & $78(46)$ & $28(40)$ & $12(43)$ & $<0.001$ \\
\hline Changes in bladder habits & $58(35)$ & $25(36)$ & $14(56)$ & $<0.001$ \\
\hline Changes in bowel habits & $73(43)$ & $25(36)$ & $12(48)$ & $<0.001$ \\
\hline Food intolerance & $93(55)$ & $38(54)$ & $16(42)$ & $<0.001$ \\
\hline Metallic taste & $39(23)$ & $15(21)$ & $12(80)$ & $<0.001$ \\
\hline Weight problems & $111(66)$ & $45(64)$ & $19(42)$ & $<0.001$ \\
\hline Thyroid disorder & $68(40)$ & $34(49)$ & $14(41)$ & $<0.001$ \\
\hline Hormone imbalance & $80(48)$ & $37(53)$ & $17(46)$ & $<0.001$ \\
\hline Early menopause & $29(17)$ & $14(20)$ & $5(36)$ & 0.063 \\
\hline Decreased libido & $116(69)$ & $53(76)$ & $22(42)$ & $<0.001$ \\
\hline Poor healing & $64(38)$ & $32(46)$ & $14(44)$ & $<0.001$ \\
\hline Fevers or night sweats & $85(51)$ & $37(53)$ & $22(59)$ & $<0.001$ \\
\hline Persistent infections & $44(26)$ & $23(33)$ & $18(78)$ & $<0.001$ \\
\hline Swollen lymph nodes & $84(50)$ & $36(51)$ & $32(89)$ & $<0.001$ \\
\hline
\end{tabular}

Values are presented as number (\%). Bll, breast implant illness.

Table 2. Characteristics of symptoms before and after explantation

\begin{tabular}{lccc}
\hline Outcome & $\begin{array}{c}\text { Explantation patients, } \\
\text { No. (\%) }\end{array}$ & $\begin{array}{c}\text { Symptoms pre-explant, } \\
\text { mean } \pm \text { SD }\end{array}$ & $\begin{array}{c}\text { Symptoms improved post-explant, } \\
\text { mean } \pm \text { SD }\end{array}$ \\
\hline Complete resolution of symptoms following explantation & $16(23)$ & $20.5 \pm 5.7$ & $18.2 \pm 6.3$ \\
Partial resolution of symptoms following explantation & $52(74)$ & $18.1 \pm 6.4$ & $10.5 \pm 5.8$ \\
No resolution of symptoms following explantation & $2(3)$ & $14.5 \pm 7.8$ & $0 \pm 0$ \\
\hline
\end{tabular}




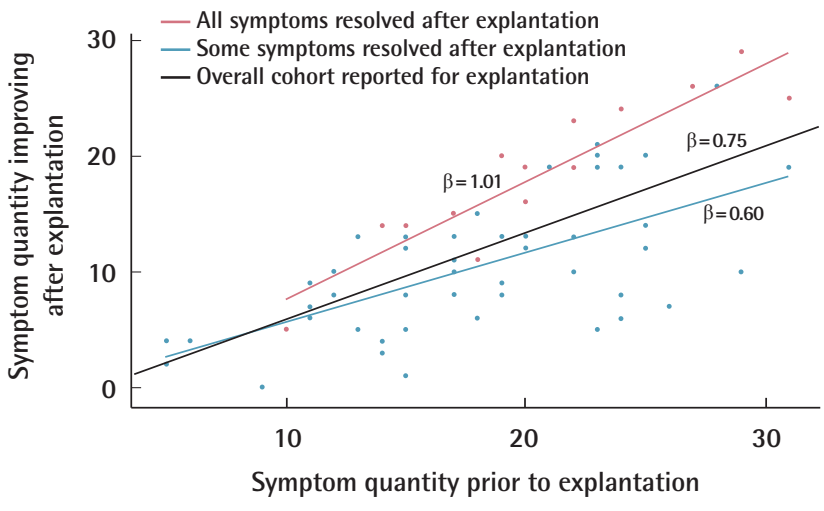

Fig. 1. Preoperative symptoms as predictor for symptom resolution. Relationship between the number of symptoms women reported prior to explantation and the number of symptoms they reported to have improved after explantation.

had the highest prevalence $(94 \%, \mathrm{n}=66)$, followed by joint pain $(83 \%, n=58)$ and hair loss $(80 \%, n=56)$. Following explantation, there was a significant improvement in all symptoms reported prior to explantation, except early menopause (Table 3 ). The symptoms that showed the greatest percent improvement following explantation were as follows: breast discomfort (91\%, $\mathrm{n}=41)$, swollen lymph nodes $(89 \%, \mathrm{n}=32)$, vertigo $(82 \%, \mathrm{n}=$ $28)$, poor sleep $(80 \%, n=37)$, headaches $(80 \%, n=35)$, metallic taste $(80 \%, n=12)$. Fatigue and brain fog had the greatest prevalence prior to explantation and reportedly improved in $76 \%(\mathrm{n}=50)$ and $73 \%(\mathrm{n}=48)$ of women following explantation, respectively (Table 3 ).

A univariate linear regression model was created to determine if an association exists between the number of BII symptoms reported by women prior to explantation and the number of symptoms that resolved after their explantation. In those women who reported a complete resolution of all their symptoms after explantation, there was a strong and direct correlation between the number of symptoms they attributed to BII prior to explantation and the number of BII symptoms that improved after their implant removal $\left(\mathrm{R}^{2}, 0.84\right)$ (Fig. 1). In those who reported only a partial resolution of their BII symptoms after implant removal, on average, for every 10 symptoms reported preoperatively, there was an improvement of six symptoms following explantation (B-coefficient, 0.60; 95\% confidence interval, 0.41-0.79) (Fig. 1). When the entire explantation cohort was evaluated, for every 10 symptoms reported preoperatively, on average, we saw 7.5 of these symptoms resolve postoperatively (B-coefficient, 0.75; 95\% confidence interval, 0.56-0.93) (Fig. 1).

A total of $64 \%(n=112)$ of survey respondents did not undergo explantation. The most frequently cited reasons for not pursuing explantation were cost (64\%), finding a surgeon (40\%), and cosmetic concerns (30\%) (Table 4).
Table 4. Reasons for not explanting

\begin{tabular}{lc}
\hline Variable & No. (\%) \\
\hline Cost & $72(64)$ \\
Poor health & $9(8)$ \\
Recovery time & $28(25)$ \\
Finding a surgeon & $45(40)$ \\
Breast appearance following explantation & $34(30)$ \\
Others & $4(4)$ \\
\hline
\end{tabular}

\section{DISCUSSION}

Systemic signs and symptoms reportedly stemming from an individual's breast implants are known today as BII. The exact mechanism by which the implant may induce BII is uncertain. Magnusson et al. [2] suggest that implant-related diseases such as BII, capsular contracture, and breast implant-associated anaplastic large cell lymphoma are a result of implant-induced inflammation. It is speculated that the presence of an antigen that is bacterially-driven, from the breakdown and leaching of the implant material, or metal remnants from the production process, leads to the exaggeration of both the cellular and humoral immune responses [17].

$\mathrm{BII}$ is a term popularized by social media in recent years, and it exemplifies the pervasive and impactful nature that such platforms can impart [1]. Several Facebook support groups for BII were evaluated in 2017 , revealing that $48 \%$ and $45 \%$ of comments focused on BII symptoms and explantation as a treatment for BII, respectively [18]. There is a large body of literature investigating the possible adverse effects of breast implants on women's health, yet no clear association has been established. Despite this, women continue to report symptoms and seek explantation, all while sharing these experiences on such platforms $[1,18,19]$.

The advent and subsequent rise of social media have been astonishing. $5 \%$ of US adults used social media in 2005 , later rising to $79 \%$ in 2019 . While $18 \%$ of adults search for others who share common health interests or experiences, $72 \%$ of US adult internet users will use the internet to obtain health information [20-22]. Even though studies have deemed that up to $90 \%$ of such content is of poor quality, patients often rely on this information to make health-related decisions [22]. This is consistent in our responses, where the internet or social media were among the most common sources for obtaining information on BII.

Social media was used to gain further insight into the opinions and experiences of women with BII through a 20-question survey distributed to the "UTAH Breast Implant Illness" Facebook group in order to better characterize the disease and its symptoms, while also investigating the outcomes following explantation. The majority of women attributed their implant-related 
health concerns to BII. There was a wide range of reported symptoms, prompting over $40 \%$ of women to undergo explantation. The majority of these women reported improvement in their symptoms. Seventy-four percent had partial improvement, while $23 \%$ reported complete resolution following explantation. Almost all symptoms reported preoperatively by women who underwent explantation showed statistically significant improvement following surgery.

The FDA's moratorium on silicone breast implants was lifted in 2006 after multiple publications were unable to provide evidence of silicone implants causing specific symptoms or diseases [8-10]. Coroneos et al. [12] published the largest analyses of long-term outcomes data from implant manufacturers in 2018 and reported a greater risk of developing specific rheumatic and connective tissue diseases when compared to the general population, leading to the resurgence of BII in the media and public. However, these conclusions were heavily critiqued by the FDA and other authors, pointing out the heterogeneity and flaws in data collection and representation, respectively, without properly addressing how sources of bias may have impacted their conclusions [14,15].

Our survey respondents who self-reported having BII describe 32 different symptoms, 19 of which had a prevalence of $>50 \%$. Among the most common were brain fog, fatigue, joint pain, and hair loss. These responses are consistent with prior literature [10,23-27]. In 2017, Cohen Tervaert and colleagues published both a prospective study and a literature review discussing the self-reported symptoms of women. They described fatigue, joint pain, and cognitive impairment as the most common symptoms [24,27]. A systematic review by Lipworth et al. [9] reported similar findings.

Rohrich et al. [28] prospectively evaluated women requesting explantation and found lower feelings of self-reported general health and physical functioning along with a higher prevalence of musculoskeletal symptoms when compared to the control population. The similarities in symptoms between our study and prior literature help increase the generalizability of our results while heightening our confidence that we are evaluating a similar entity to what other authors are studying in the United States and Europe.

Women present for explantation in order to improve their symptoms, often at the recommendation of other women $[1,18]$. Just over $40 \%$ of respondents that reported BII underwent explantation. Most importantly, almost all women self-reported partial or complete resolution of symptoms, with only two women denying improvement following explantation.

In Rohrich's analysis of explantation outcomes, he revealed a significant improvement in overall general and mental health
[28]. This was supported by prospective study and literature review of Cohen Tervaert and colleagues, where $50 \%$ and $75 \%$ of women reported improvement following explantation, respectively [24,27]. Peters et al. [25] revealed differences between three groups of women regarding outcomes following explantation. Women without a diagnosis of autoimmune or rheumatologic conditions had substantial improvements in their physical and psychological health, while no improvement and transient improvement were reported by those women with a diagnosed autoimmune disease and rheumatologic disease, respectively.

Our study also revealed symptomatic improvement following explantation. Unique to our analysis is the demonstration of statistically significant improvements in these symptoms. We also demonstrate that, in general, the more symptoms a patient has preoperatively, the more symptoms that will resolve postoperatively. This suggests that implant explantation does not only treat a subset of symptoms.

As physicians, we should not discount the concern women have with their implants. A review of social media comments regarding BII describes women's discontent with their plastic surgeon and dismissal of their complaints [18]. This dismissal will undoubtedly lead some women to seek advice from non-medical professionals, which may, in turn, lead to misconceptions of what is encompassed by BII. Well-documented complications exist with cosmetic breast augmentation, including surgical site complications, implant rupture, and capsular contraction. However, these are complications unrelated to the current understanding of BII. Furthermore, following the FDA's recent acknowledgment of breast implant-associated anaplastic large cell lymphoma, there has been a substantial and concurrent rise of search queries and tweets on BII and its relationship to cancer, which reveals the extent of conditions women are associating with BII [29].

Identifying misconceptions is vital to our patients' care so that we can provide them with updated and accurate information regarding the differences between BII and other entities; this is only plausible if we convey an atmosphere of understanding and trust. Regarding explantation, McGuire et al. [19] remind us that patients reserve the same right to have their implants removed as they had when they sought augmentation. It is our responsibility to ensure their understanding of both the possible benefits and complications that explantation may bring, which is ultimately hindered if women look elsewhere for information and support.

Limitations exist in our work. The distribution of the survey to only those involved with Utah BII social media group introduces a level of responder bias to our results. The survey's anonymous nature also made it difficult to control for the possibility 
of fake profiles and did not allow data verification. The lack of a control group will limit the conclusions we may draw regarding the differences in the prevalence of symptoms between BII patients and those without BII. Furthermore, the generalizability of our results to other women reporting BII will be limited in several ways. First, members of this group represent only a fraction of women with breast implants in Utah. Second, we received responses from only a sample of members. However, considering Utah's substantial rate of cosmetic surgery, plastic surgeons per capita, and its predominantly white population, we believe that the population captured in our study is representative of the cosmetic surgery population in the United States $[3,30]$. Lastly, due to privacy constraints, the authors could not perform a nonresponder analysis that may sometimes be performed when survey response rates are low to ensure the results represent the population of interest. Although the survey received an unweighted response rate of $14.2 \%$, we believe this rate to be effectively higher than reported. Given the nature of social media, there will be varying levels of involvement and participation in such groups. In turn, it is highly likely that only a fraction of members were exposed to the survey, effectively decreasing the overall number of members, which would ultimately increase our response rate. Considering this group consists of members who self-identify as having BII, and also includes members who have not undergone explantation, the authors believe that our response rate is adequate to represent the broader population of interest.

Certain patient demographics and characteristics were not extracted from our survey. Knowing a patient's age, for example, can give insight into what symptoms could better correlate with aging rather than implants. Past medical history would give better insight into a patient's current state of overall physical and mental health and how it could contribute to their symptoms. Half of our cohort reported an autoimmune disease, the majority of which reported onset following implantation. However, it is unknown if these diagnoses were established by a medical provider or self-reported. Thus, knowing a respondent's family history could give insight into a genetic predisposition for developing these conditions or symptoms.

Lastly, we found that the overwhelming majority of patients' symptoms began greater than 2 years after implantation. Determining a more accurate timeline between implant exposure and explantation to the development and resolution of their symptoms, respectively, may better estimate the length of time it takes for women to develop symptoms after implantation and the time it takes for symptoms to improve following explantation. Future studies are needed to understand the temporal relationship between the placement of breast implants and the onset of

\section{BII symptoms.}

A survey was distributed to the Utah BII social media group receiving 182 total responses. An extensive list of symptoms attributed to BII was reported by women, leading some to seek explantation. The four most common symptoms reported were brain fog (decreased ability to concentrate), fatigue, joint pain, and hair loss. All but two women described either partial or complete symptom resolution following explantation. Furthermore, we revealed an association between the number of symptoms improved after explanation and the number of symptoms reported preoperatively. Our results provide a better characterization of how patients report their BII symptoms as well as patient-reported outcomes following explantation. We believe our research will serve as a stepping-stone for future work involving larger cohorts of women reporting BII.

\section{NOTES}

\section{Conflict of interest}

Jayant P. Agarwal is a consultant for DonJoy Orthopedics. The rest of the authors have nothing to disclose.

\section{Ethical approval}

The survey and study design were reviewed by the Review Board of the University of Utah Hospital and were given exempt status. Informed consent was waived by an anonymous online survey.

\section{Author contribution}

Conceptualization: TC Jessop, JW Garlick, JS Manum, JP Agarwal, AC Kwok. Data curation: TC Jessop, GC Carter. Formal analysis: GC Carter. Methodology: TC Jessop, JW Garlick, JS Manum, GC Carter, JP Agarwal, A Kwok. Project administration: TC Jessop, JW Garlick, JS Manum, JP Agarwal, A Kwok. Visualization: DA Magno-Padron, JP Agarwal, A Kwok. Writing - original draft: DA Magno-Padron, JW Garlick, GC Carter. Writing - review \& editing: DA Magno-Padron, J Luo, TC Jessop, JW Garlick, GC Carter, JP Agarwal, A Kwok.

\section{ORCID}

David A. Magno-Padron

$\begin{array}{ll} & \text { https://orcid.org/0000-0001-6577-2413 } \\ \text { Jessica Luo } & \text { https://orcid.org/0000-0003-2673-745X } \\ \text { Terry C. Jessop } & \text { https://orcid.org/0000-0001-9948-9552 } \\ \text { Jared W. Garlick } & \text { https://orcid.org/0000-0002-9686-422X } \\ \text { Joanna S. Manum } & \text { https://orcid.org/0000-0001-7071-9297 } \\ \text { Gentry C. Carter } & \text { https://orcid.org/0000-0001-7175-3964 } \\ \text { Jayant P. Agarwal } & \text { https://orcid.org/0000-0002-1209-6703 }\end{array}$


Alvin C. Kwok https://orcid.org/0000-0003-2215-3764

\section{Supplementary material}

Supplemental Material 1. Breast implant illness survey. Supplemental data can be found at: https://doi.org/10.5999/aps.2020.02117

\section{REFERENCES}

1. Healing Breast Implant Illness [Internet]. Bowser, BC: Healing Breast Implant Illness [cited 2019 Nov 1]. Available from: https://healingbreastimplantillness.com.

2. Magnusson MR, Cooter RD, Rakhorst H, et al. Breast implant illness: a way forward. Plast Reconstr Surg 2019; 143(3S A Review of Breast Implant-Associated Anaplastic Large Cell Lymphoma):74S-81S.

3. American Society of Plastic Surgeons (ASPS). Plastic surgery statistics report [Internet]. Arlington Heights, IL: ASPS; c2018 [cited 2019 Nov 1]. Available from: https:// www.plasticsurgery.org/documents/News/Statistics/2018/ plastic-surgery-statistics-full-report-2018.pdf.

4. Vera-Lastra O, Medina G, Cruz-Dominguez Mdel P, et al. Human adjuvant disease induced by foreign substances: a new model of ASIA (Shoenfeld's syndrome). Lupus 2012; 21:128-35.

5. Miyoshi K, Miyaoka T, Kobayashi Y, et al. Hypergammaglobulinemia by prolonged adjuvanticity in man: disorders developed after augmentation mammoplasty. Jpn Med J 1964;2122:9-14.

6. Kessler DA. The basis of the FDA's decision on breast implants. N Engl J Med 1992;326:1713-5.

7. Sergott TJ, Limoli JP, Baldwin CM Jr, et al. Human adjuvant disease, possible autoimmune disease after silicone implantation: a review of the literature, case studies, and speculation for the future. Plast Reconstr Surg 1986;78:104-14.

8. Janowsky EC, Kupper LL, Hulka BS. Meta-analyses of the relation between silicone breast implants and the risk of connective-tissue diseases. N Engl J Med 2000;342:781-90.

9. Lipworth L, Tarone RE, McLaughlin JK. Silicone breast implants and connective tissue disease: an updated review of the epidemiologic evidence. Ann Plast Surg 2004;52:598601.

10. Tugwell P, Wells G, Peterson J, et al. Do silicone breast implants cause rheumatologic disorders? A systematic review for a court-appointed national science panel. Arthritis Rheum 2001;44:2477-84.

11. Center for Devices and Radiological Health U.S. Food and Drug Administration. FDA update on the safety of silicone gel-filled breast implants. Silver Spring: U.S. Food and Drug
Administration; 2011.

12. Coroneos CJ, Selber JC, Offodile AC 2nd, et al. US FDA breast implant postapproval studies: long-term outcomes in 99,993 patients. Ann Surg 2019;269:30-6.

13. Rohrich RJ, Kaplan J, Dayan E. Silicone implant illness: science versus myth? Plast Reconstr Surg 2019;144:98-109.

14. Nava MB, Catanuto G, Rocco N. Comment on "US FDA Breast Implant Post Approval Studies. Longterm Outcomes in 99,993 Patients”. Ann Surg 2019;270:e63.

15. Ashar B. Statement from Binita Ashar, M.D., of the FDA's Center for Devices and Radiological Health on agency's commitment to studying breast implant safety. Silver Spring: U.S. Food and Drug Administration; 2018.

16. UTAH Breast Implant Illness [Internet]. c2016 [cited 2020 Jan 12]. Available from: https://www.facebook.com/ groups/1215360765175534/.

17. Lee M, Ponraja G, McLeod K, et al. Breast implant illness: a biofilm hypothesis. Plast Reconstr Surg Glob Open 2020; 8:e2755.

18. Tang SY, Israel JS, Afifi AM. Breast implant illness: symptoms, patient concerns, and the power of social media. Plast Reconstr Surg 2017;140:765e-766e.

19. Mcguire PA, Haws MJ, Nahai F. Breast implant illness: how can we help? Aesthet Surg J 2019;39:1260-3.

20. Ortiz-Ospina E. The rise of social media [Internet]. Oxford: Our World in Data; c2019 [cited 2019 Nov 1]. Available from: https://ourworldindata.org/rise-of-social-media.

21. Pew Research Center. Peer-to-peer Health Care [Internet]. Washington, D.C.: Pew Research Center; c2011 [cited 2019 Nov 1]. Available from: https://www.pewresearch. org/internet/2011/02/28/peer-to-peer-health-care-2/.

22. Montemurro P, Porcnik A, Heden P, et al. The influence of social media and easily accessible online information on the aesthetic plastic surgery practice: literature review and our own experience. Aesthetic Plast Surg 2015;39:270-7.

23. Cohen Tervaert JW, Kappel RM. Silicone implant incompatibility syndrome (SIIS): a frequent cause of ASIA (Shoenfeld's syndrome). Immunol Res 2013;56:293-8.

24. Colaris MJ, de Boer M, van der Hulst RR, et al. Two hundreds cases of ASIA syndrome following silicone implants: a comparative study of 30 years and a review of current literature. Immunol Res 2017;65:120-8.

25. Peters W, Smith D, Fornasier V, et al. An outcome analysis of 100 women after explantation of silicone gel breast implants. Ann Plast Surg 1997;39:9-19.

26. Breiting VB, Holmich LR, Brandt B, et al. Long-term health status of Danish women with silicone breast implants. Plast Reconstr Surg 2004;114:217-28. 
27. de Boer M, Colaris M, van der Hulst RR, et al. Is explantation of silicone breast implants useful in patients with complaints? Immunol Res 2017;65:25-36.

28. Rohrich RJ, Kenkel JM, Adams WP, et al. A prospective analysis of patients undergoing silicone breast implant explantation. Plast Reconstr Surg 2000; 105:2529-43.

29. Adidharma W, Latack KR, Colohan SM, et al. Breast im- plant illness: are social media and the internet worrying patients sick? Plast Reconstr Surg 2020;145:225e-227e.

30. Nielsen L. Utah No. 6 in US for number of plastic surgeons per capita [Internet]. Salt Lake City, UT: KSL News; c2017 [cited 2020 Jan 5]. Available from: https://www.ksl.com/ article/43505715/utah-no-6-in-us-for-number-of-plasticsurgeons-per-capita. 